\title{
Increasing Physical Activity Among African-American Women and Girls
}

Melicia C. Whitt-Glover

Dorine J. Brand

Maren E. Turner

Erica M. Jackson

William \& Mary

Follow this and additional works at: https://scholarworks.wm.edu/aspubs

\section{Recommended Citation}

Whitt-Glover, M. C., Brand, D. J., Turner, M. E., Ward, S. A., \& Jackson, E. M. (2009). Increasing physical activity among African-American women and girls. Current sports medicine reports, 8(6), 318-324.

This Article is brought to you for free and open access by the Arts and Sciences at W\&M ScholarWorks. It has been accepted for inclusion in Arts \& Sciences Articles by an authorized administrator of W\&M ScholarWorks. For more information, please contact scholarworks@wm.edu. 


\title{
Increasing Physical Activity Among African-American Women and Girls
}

\author{
Melicia C. Whitt-Glover, ${ }^{1}$ Dorine J. Brand, ${ }^{2}$ Maren E. Turner, ${ }^{3}$ Sheila A. Ward, ${ }^{4}$ and Erica M. Jackson ${ }^{5}$ \\ ${ }^{1}$ Gramercy Research Group, Winston Salem, NC; ${ }^{2}$ University of Illinois at Urbana-Champaign, Department of \\ Kinesiology and Community Health, Champaign, IL; ${ }^{3}$ University of Kansas, Department of Applied Behavioral \\ Science, Lawrence, KS; ${ }^{4}$ Department of Health, Physical Education, and Exercise Science, Norfolk State University, \\ Norfolk, VA; ${ }^{5}$ College of William \& Mary, Department of Kinesiology \& Health Sciences, Williamsburg, VA
}

WHITT-GLOVER, M.C., D.J. BRAND, M.E. TURNER, S.A. WARD, and E.M. JACKSON. Increasing physical activity among African-American women and girls. Curr. Sports Med. Rep., Vol. 8, No. 6, pp. 318-324, 2009. The benefits of physical activity on diseases and risk factors are well known. Despite the known benefits, many segments of the population, particularly African-American women and girls, do not obtain adequate levels of physical activity. Strategies are needed to identify successful and sustainable interventions to increase physical activity among this population. We reviewed literature published between 2007 and 2009 that focused on increasing physical activity or fitness among this population. We identified 37 studies, 11 of which focused on increasing physical activity. This article summarizes the findings from those 11 studies and provides recommendations for improving strategies to increase physical activity in African-American women and girls.

\section{INTRODUCTION}

The benefits of increased physical activity on diseases and risk factors such as cardiovascular disease, hypertension, diabetes, obesity, and some forms of cancer, particularly among racial/ethnic minorities and women, have been well documented $(9,17,30,40)$. Despite the known benefits, physical activity levels among racial and ethnic minorities and women, including African-American women, remain low. The trend for low physical activity among AfricanAmerican girls and women actually begins in childhood and early adolescence. For example, Kimm and colleagues reported on data collected in the National Heart, Lung, and Blood Institute's Growth and Health Study (NGHS) that included data from 2379 black and white girls (20). Participants were followed from ages $9-10$ yr to $18-19$ yr. At baseline, the self-reported median activity scores (metabolic equivalent [MET]-times' $\mathrm{wk}^{-1}$ ) were 27.3 and 30.8 for black and white girls, respectively. After $10 \mathrm{yr}$, physical activity scores declined to 0 and $11.0 \mathrm{MET}$-times' $\mathrm{wk}^{-1}$ for black and white girls, respectively, representing a 100\% de-

Address for correspondence: Melicia C. Whitt-Glover, Ph.D., FACSM, Gramercy Research Group, 500 West 4th St., Suite 203, Winston Salem, NC 27101

(E-mail: mwhittglover@gramercyresearch.com).

1537-890X/0806/318-324

Current Sports Medicine Reports

Copyright (C) 2009 by the American College of Sports Medicine cline in reported physical activity for black girls. National surveillance data for adults shows that the trend for lower levels of physical activity among African-American women continues throughout adulthood. Comparisons among adults show lower levels of physical activity among African-American people compared with white people, with the lowest levels reported among African-American women (8,29). Estimates from national surveillance data consistently show that only $24 \%-36 \%$ of African-American people participate in regular physical activity, defined as at least $30 \mathrm{~min}$ of moderateintensity physical activity on at least $5 \mathrm{~d} \cdot \mathrm{wk}^{-1}$ or at least $20 \mathrm{~min}$ of vigorous-intensity physical activity on at least $3 \mathrm{~d} \cdot \mathrm{wk}^{-1}$ (43). National surveillance data also consistently show that physical activity participation is lowest among African-American women compared with men, regardless of age, education, income level, marital status, employment status, or region in which respondents reside. Similar findings have been observed in smaller, regional and local datasets using self-reported and objectively-assessed physical activity data $(1,6,46-47)$.

The consequences of low physical activity levels, particularly among African-American women, are evident in the high prevalence rates of poor health outcomes associated with low levels of physical activity. National datasets consistently show higher rates of chronic diseases, such as diabetes and hypertension, and risk factors such as obesity among African-American women compared with AfricanAmerican men and compared with men and women from other racial and ethnic groups $(7,33)$. Data from the NGHS 
show that the trend for poor health outcomes associated with low levels of physical activity among African-American girls compared with white girls begins in childhood and early adolescence, and the data have demonstrated a higher prevalence of obesity, insulin resistance and impaired fasting glucose, and other risk factors associated with the development of cardiovascular disease among African-American girls compared with white girls $(19,23,31)$. Successful and sustainable strategies are needed to increase physical activity participation levels among African-American women and girls, which could help to address disparities in health outcomes associated with low levels of physical activity.

A recently published review of the literature identified 43 intervention studies published between 1985 and 2006 focused on increasing physical activity or fitness among African-American people (45). The review also identified characteristics of effective interventions and provided general conclusions and suggestions for future interventions to increase physical activity among African-American people. The current topic review extends findings from the previous review, summarizes articles published from 2007 through 2009 regarding African-American women and girls, and offers implications and additional suggestions for future interventions.

\section{METHODS}

Potential articles to be included in the current topic review were identified through a search of the PubMed database (available at www.ncbi.nlm.nih.gov/pubmed/). Search terms included black, African-American, ethnic, minority, intervention, program, exercise, physical activity, fitness, training, health education, physical fitness, physical training, and weight loss. Reference lists for identified articles also were used to identify additional studies. No attempt was made to search unpublished literature or thesis/dissertation projects or to search databases other than PubMed because previous experience with a similar literature review identified very few additional articles using those sources (45).

Articles were included in the current topic review if they met the following three criteria: 1) they were published between 2007 and 2009, described an intervention, and listed increased physical activity as an outcome of interest or as a component of the intervention; 2) they reported physical activity assessment or physical fitness outcome data; and 3) they included African-American women and girls and presented physical activity outcome data separately for African-American or black participants. We also accepted studies that did not present data separately for AfricanAmerican women if the percentage of African-American women included in the study population was $\geq 85 \%$.

Two authors (M.C. Whitt-Glover and M.E. Turner) conducted separate searches using the PubMed database and identified potential articles of interest based on abstracts. Once potential studies were identified, one author (D.J. Brand) distributed copies of articles to three authors (M.C. WhittGlover, M.E. Turner, and D.J. Brand) for review and data extraction. For each article identified that met criteria for inclusion, the following information was recorded using a standardized study extraction form: 1) publication citation; 2) study objective; 3) study design and length; 4) study setting; 5) description of study participants, including sample size, life stage, and health status; 6) participant recruitment strategies; 7) description of intervention and control conditions, including the underlying theories used to develop the intervention; 8) specific strategies for cultural adaptation within the study; 9) specific strategies used to increase physical activity; 10) main outcomes of interest for the study and how outcomes were assessed; 11) physical activity-related outcomes of interest and how outcomes were assessed if physical activity was not the main outcome; and 12) effect of the intervention strategies on the main outcome and on physical activity.

\section{RESULTS}

We identified 37 studies that potentially met inclusion/ exclusion criteria; however, after further review and data extraction, only 11 studies focused on change in physical activity or cardiovascular fitness as one of the primary endpoints. Table 1 presents a summary of the findings from the studies included in the current topic review. Three studies focused on girls or adolescents, and the others focused on African-American women. The age range for participants in the adult studies varied widely. Most studies were conducted in community-based settings - only one study was conducted in a clinical setting (14). Most studies were short-term, lasting a few weeks to 6 months; however, one after-school study among African-American girls lasted for 10 months (5), and one community-based study among African-American women lasted 12 months (4). Most studies collected post-intervention data immediately at the end of the intervention with no further follow-up. Only one study collected additional data 6 months after the intervention ended (4). One additional community-wide study collected data $4 \mathrm{yr}$ after the intervention, based on when a community-wide survey already was scheduled to be administered (35).

Our previous review of the literature focused on interventions for increasing physical activity in African-American communities and ranked studies on several factors, including study design, whether physical activity goals for participants were specified, and physical activity assessment methods (45). Although we did not rank studies in the current topic review, we note that 5 of the 11 studies were randomized, controlled trials, which is considered the most scientifically rigorous method for testing a hypothesis and the strongest study design for determining intervention efficacy $(24,26)$.

Four of the studies included in the current topic review provided participants with specific goals for physical activity that were in line with nationally recognized recommendations for moderate-intensity physical activity $\left(\geq 30 \mathrm{~min} \cdot \mathrm{d}^{-1}\right.$; $\left.\geq 150 \mathrm{~min}^{-\mathrm{wk}^{-1}}\right)$ or for daily walking $\left(10,000 \mathrm{steps}^{-1}\right.$ ) $(10,14,39,44)$. One study encouraged participants to obtain an additional 10,000 steps $\mathrm{wk}^{-1}$ above current walking levels, which, while not in line with any empirically determined physical activity recommendation, was at least a goal that could be measured (18). Three studies offered physical activity goals for participants that could be considered vague, such as to walk with a partner at least $2 \mathrm{~d} \cdot \mathrm{wk}^{-1}$ 
TABLE 1. Summary of studies focused on increasing physical activity in African-American individals published between January 2007 and June 2009.

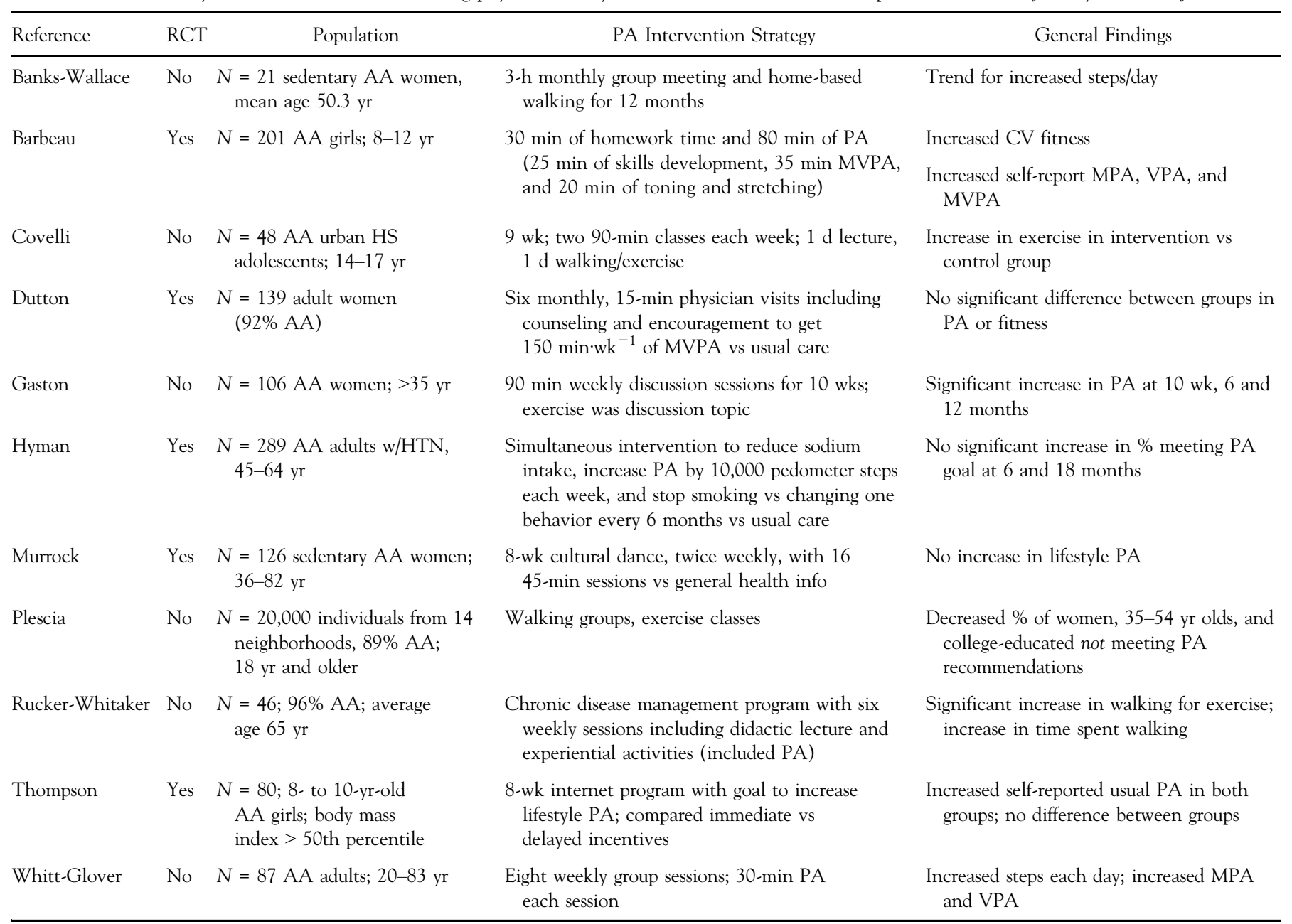

$\mathrm{AA}=$ African-American, $\mathrm{CV}=$ cardiovascular fitness, HS = high school, HTN = hypertension, MPA = moderate-intensity physical activity, MVPA = moderate-to-vigorous intensity physical activity, $\mathrm{PA}=$ physical activity, $\mathrm{RCT}=$ randomized controlled trial, $\mathrm{VPA}=$ vigorous-intensity physical activity.

(4), exercise more (15), and increase lifestyle physical activity (39). Four other studies included in the current topic review did not specify physical activity goals for participants $(5,32,35,37)$.

Studies included in the current topic review used a variety of methods for assessing physical activity including pedometers (four studies), fitness tests (two studies), and self-report measures (nine studies). Several studies used more than one method to assess physical activity. In general, studies showed changes in physical activity in the expected direction. Eight of the 11 studies included in this topic review reported positive changes in daily walking, fitness, or self-reported physical activity; however, most of the studies reported these changes immediately post-intervention and the extent to which these changes impacted long-term behavior (e.g., $>24$ months after the end of the intervention) is unclear.

\section{DISCUSSION AND RECOMMENDATIONS}

The current topic review identified 11 studies focused on increasing physical activity in African-American women and girls and published between January 2007 and June 2009. A summary of potential intervention strategies and additional research needs, based on the current topic review, is included in Table 2. In general, studies showed positive changes in physical activity. Findings from the current topic review are similar to findings from previous reviews of physical activity interventions in racial/ethnic minority groups that also showed positive changes in physical activity levels immediately after interventions $(3,38,45)$. Most of the studies included in this topic review assessed physical activity using self-report methods, which may be subject to recall bias or social desirability bias. If at all possible, objective measures of physical activity (e.g., pedometers, accelerometers/motion detectors) are recommended. We recognize, however, that there are also potential issues associated with objective measures of physical activity. For example, there is potential for pedometers to act as an intervention, particularly among control group participants in controlled trials, because pedometers may increase awareness of physical activity levels and may motivate participants spontaneously to increase physical activity levels. This is entirely possible, particularly since many studies use pedometers as a motivational tool in 
interventions designed to increase physical activity. Given the publicity around increasing physical activity in the lay media, many individuals are aware of the 10,000 steps $\cdot \mathrm{d}^{-1}$ recommendation (27). In studies where pedometers are used to assess physical activity and, particularly among control groups, investigators may wish to blind participants from looking at daily step counts and altering their physical activity by applying tamper-proof tape to prevent the pedometer from being opened. It is unlikely that most individuals are sufficiently motivated to alter physical activity levels on their own to impact study findings substantially, except in cases of highly motivated individuals. However, investigators may wish to consider a "run-in" period during which study participants wear a pedometer and monitor physical activity levels for 2-3 d prior to the start of official data collection. The "run-in" period may provide time for the novelty of a new gadget to wear off, thus allowing collection of data on true physical activity levels.

Potential issues with using accelerometers for physical activity data collection include adherence to monitoring protocols to allow complete data collection and the use of algorithms that can appropriately convert accelerometer counts to accurate assessments of energy expenditure and physical activity. This has particularly been an issue among overweight and obese participants who may have difficulty with monitor placement and for whom current accelerometer algorithms may not be appropriate for estimating energy expenditure levels. To discuss issues associated with accelerometer assessment, two expert panels, funded by the National Institutes of Health, have been convened. One supplement to Medicine $\mathbb{E}$ Science in Sports $\mathbb{E}$ Exercise $_{\mathbb{B}}$ has been published to address these issues (2005, Supplement 11), and one additional supplement is forthcoming in Medicine $\mathbb{E}$ Science in Sports $\mathcal{B}$ Exercise Ex $_{\circledast}$ in 2010.

In addition to using self-report methods, most studies included in the current topic review assessed physical activity immediately after the intervention ended. The $1998 \mathrm{Na}$ tional Heart, Lung, and Blood Institute (NHLBI) conference on "Maintenance of Behavior Change in Cardiorespiratory Risk Reduction" highlighted issues related to adoption and maintenance of physical activity as well as cross-cutting themes in the maintenance of behavior change from research focusing on other behaviors (e.g., smoking) and two key physical activity studies that included follow-up through 24 months and provided insight on factors associated with behavior change maintenance $(28,34,49)$. Although the NHLBI conference occurred in 1998, it is evident from the current topic review that there are still a limited number of studies that have looked at long-term maintenance of increased levels of physical activity (e.g., $\geq 24$ months postintervention) (11-13,21-22). Additional research is needed to highlight the long-term sustainability of intervention strategies.

Although we initially identified 37 studies for the current topic review, only 11 studies focused on increasing physical activity solely or in conjunction with improving other behaviors. Findings from the current topic review confirm findings from previous reviews - most published interventions involving increasing physical activity among AfricanAmerican women and girls do not focus solely on physical activity. There is some speculation that focusing on changing multiple behaviors at the same time may be difficult and overwhelming for study participants. One study included in this topic review tested the impact of an intervention to change three behaviors simultaneously (increased physical activity, reduced sodium intake, and smoking cessation) compared with focusing on changing the behaviors individually. Data were collected at 6-month intervals, and intervention strategies were compared with a no-treatment control group (18). The findings showed nonsignificant increases in the percentage of participants meeting physical activity goals, although there was a trend toward greater increases in the group making multiple changes compared with other groups. Additional research may be needed to determine whether it is more useful to focus on a single behavior or on multiple behaviors simultaneously. Additional research also may be needed to determine whether focus on multiple behaviors simultaneously depends on the behaviors (e.g., focus on diet and physical activity simultaneously may be a more natural combination that focusing on diet and smoking cessation simultaneously).

Numerous recommendations exist that outline the suggested amount of physical activity recommended to impact outcomes of interest (e.g., blood pressure, weight loss). Although not presented in the current review of the literature, many studies have historically used physical activity/exercise as an intervention strategy to impact these outcomes of interest. Often, studies show little or no change in the outcomes of interest as a result of the physical activity interventions. Unfortunately, as was the case with studies included in the current topic review, the extent to which participants actually achieved the recommended level of physical activity is often unclear (i.e., did study participants actually achieve and maintain the level of physical activity necessary to achieve the desired outcome?). Few studies, including those presented in the current topic review, actually report adherence in terms of achieving study-specific physical activity recommendations. Recommending the appropriate level of physical activity in the intervention and then assessing the extent to which participants adhere to the recommendation would be useful for determining 1) whether the intervention strategies were effective in increasing physical activity and 2) whether the prescribed level of physical activity was sufficient to impact the outcome of interest. Information about the impact of prescribed levels of physical activity on outcomes of interest would be useful to help interventionists/health educators identify the exact amount of physical activity to prescribe, particularly in large-scale and/or community-based health education/health behavior programs.

Our previous review of the literature before 2007 on physical activity interventions for African-American individuals suggests that more research is needed to determine whether cultural adaptations to physical activity interventions actually affect outcomes (45). Methods typically used in an attempt to adapt intervention programs culturally include recruiting all African-American participants, matching group leaders by race/ethnicity and/or gender, using specific types of music that might resonate more with the target population (e.g., gospel music), and using cultural 
TABLE 2. Summary of recommendations.

Potential Intervention Strategies

Use objective measures of physical activity where possible.

When appropriate, blind participants from looking at physical activity levels (e.g., step counts on a pedometer) to prevent participants from increasing physical activity levels as a result of awareness of being monitored.

Consider using a "run-in" period during which participants monitor physical activity levels for $2-3 \mathrm{~d}$ before the start of official data collection.

Carefully assess the extent to which participants actually adhere to study-specific physical activity recommendations during interventions.

Consider incorporating African-American culture and history, as it relates to physical activity, as a method for changing attitudes and beliefs associated with physical activity among African-American women and girls.

Consider providing participant-specific information about the benefits of increased physical activity on health and expected short- and long-term benefits of physical activity.

Consider providing opportunities for participants to rehearse (and re-rehearse) the desired physical activity intensity level and bout duration in the presence of project staff before asking participants to engage in activities on their own.

Additional Research Needs

Additional research is needed to highlight the long-term (e.g., $\geq 24$ months post-intervention) sustainability of intervention strategies.

Additional research is needed to determine whether interventions focused on one behavior at a time are more/less successful than interventions focused on several behaviors at once. Additional research also is needed to determine whether the success of focusing on several behaviors simultaneously is dependent on the behaviors of focus.

Additional research is needed to determine the extent to which cultural adaptations (compared with unadapted) physical activity interventions among African-American girls and women are necessary for interventions to be acceptable and to impact outcomes of interest.

Additional testing is needed to determine short- and long-term effects of strategies to incorporate physical activity into organizational practices (e.g., during workday, at sporting events).

Additional research may be needed to determine the simultaneous effects of behavioral interventions and strategies to alter the social and built environment on physical activity levels

dance. Several studies included in the current topic review used the aforementioned methods to adapt interventions culturally, but again, because these adaptations were attempted as a part of several strategies to increase physical activity, the specific impact of the culturally adapted component of the program was unclear.

Anecdotally, African-American researchers have noted that programs that incorporate African-American culture and history may be useful for changing attitudes and beliefs related to physical activity. Future interventions designed to increase physical activity among African-American females may consider including a knowledge-based component that details the historical and cultural perspective on the role of physical activity throughout the lives of African-American people. This component may provide insight into presentday attitudes toward physical activity by exploring the complex relationship of physical activity in the lives of African-American people, where often the negative experiences of physical activity overshadow the positive experiences. For example, this broader cultural perspective might include that "during one of the lowest periods in American history, characterized by forced labor and other degrading acts, African-American individuals still chose to be physically active" (41). This reflection on history could reveal that African-American people are inherently physically active, and they once "highly prioritized positive, cultural, physical activity experiences," and it could provide empowerment for African-American females to reclaim that physically active lifestyle (41). Additionally, a historical and cultural perspective to physical activity provides participants the opportunity to value the intent and purpose of movement or physical activity and contemplate how physical activity once again can be enjoyed as an integral part of everyday life (42).

It is interesting to note that the majority of the interventions in the current review focused on asking participants to adopt an exercise program, usually in addition to their regular activities. This is inherently problematic since lack of time is listed as one of the most common barriers to physical activity. In addition, previous research has demonstrated that African-American women generally do not feel that they have free time and that, when they do have free time, rest is more important than engaging in physical activity so that one might be able to effectively engage in general daily activities (2). Understanding the benefits of physical activity also may be a critical aspect to increasing physical activity among African-American females. Exercising for chronic disease management may be viewed differently than exercising to improve or maintain health and fitness. Exercising for chronic disease management may be viewed as another activity on the "must-do" list. Even if an exercise regimen is established, it may not be maintained if individuals do not see an immediate difference in their conditions. Individuals also may be inclined to stop exercising after they attain desired effects (e.g., goal weight or desirable blood pressure, blood glucose, or cholesterol values).

Several studies have attempted to incorporate "lifestyle" physical activity into daily routines and have had limited success. One potential issue with incorporating lifestyle activity is that it typically is done in short bursts (e.g., taking the stairs instead of the elevator, parking at the back of the 
parking lot), and the bout duration of the exercise may not be sufficient to confer health benefits. More recently, Yancey et al. have begun to promote "push" strategies for increasing physical activity, whereby physical activity is incorporated into organizational practices (e.g., during half-time at sporting events, as a part of classroom-based activity in schools), making physical activity the "norm" and something that is difficult to avoid. "Push" strategies have been somewhat successful in increasing physical activity in organizations including worksites and churches $(25,48,50)$. Additional work is underway to test the impact of "push" strategies to incorporate physical activity in schools and sporting events. (www.ph.ucla.edu/cehd/activity_breaks.htm).

Previous research has indicated that personal behavior is highly influenced by a person's social (e.g., friends, family members, culture) and built (e.g., location and condition of parks, sidewalks, greenways) environments. While several of the studies included in the current review focused on changes in the social environment (e.g., asking participants to identify walking partners, in an attempt to address attitudes and norms related to physical activity), no studies incorporated concurrent changes in the built environment to support physical activity. Previous research has indicated that low-income and racial/ethnic minority communities tend to have lower access to parks and recreation facilities $(16,36)$. Thus it may be difficult, at best, to achieve increases in physical activity in resource-poor communities where individuals do not have adequate facilities to engage in physical activity. Even in interventions that promote walking, which is a low-cost option that does not require equipment or facilities, participants may encounter limitations due to the lack of or poorly maintained sidewalks, safety issues, or traffic concerns that might impact participation in walking.

Although not addressed in the studies specific to our review, it may be important for future intervention studies to provide opportunities to rehearse target behaviors in the presence of intervention leaders. Only one study included in the current topic review reported providing opportunities for participants to rehearse physical activity intensity and duration during intervention sessions (44). Anecdotally, this group of authors has experienced intervention participants not understanding prescribed intensity level and bout duration, which has impacted the participant's ability to adhere to physical activity recommendations. For example, one author (M.E. Turner) is currently conducting a study to increase physical activity among African-American women, in which participants are encouraged to engage in at least $150 \mathrm{~min} \cdot \mathrm{wk}^{-1}$ of brisk walking. Although participants were provided with written instructions with the physical activity recommendation, the investigator has had to implement several additional strategies to get women to walk at the prescribed level, including prescribing a specific number of steps to be obtained per minute and describing physical changes participants might feel while engaging in a brisk bout of walking (e.g., light sweating, increased heart rate and breathing). These strategies commonly are used in physical activity interventions; however, the investigator has discovered that few participants actually are able to translate these instructions into the appropriate exercise bout duration and intensity level. Subsequently, the investigator has had to walk with individual participants to demonstrate physical changes associated with brisk bouts of walking and has used a metronome and popular songs to assist participants with maintaining the appropriate step speed. Her experiences suggest that investigators for future studies may need to incorporate more opportunities for participants to rehearse (and re-rehearse when necessary) physical activity to be sure participants are able to adhere to the prescribed level of physical activity. In addition, participants should be given clear and practical strategies for monitoring physical activity outside the intervention setting (e.g., steps per minute set to popular songs or a metronome) to ensure adherence to physical activity protocols.

\section{CONCLUSION}

The current topic review highlights recently published studies describing interventions designed to increase physical activity among African-American women and girls. Because African-American women and girls suffer disproportionately from poor health outcomes associated with low levels of physical activity, it is important to identify successful strategies for increasing and maintaining increased levels of physical activity. The current topic review also has identified potential strategies for improving interventions to increase physical activity in African-American women and girls, as well as areas where additional research may be needed to increase the effectiveness of strategies for increasing physical activity levels among African-American women and girls.

\section{References}

1. Adams-Campbell LL, Rosenberg L, Washburn RA, et al. Descriptive epidemiology of physical activity in African-American women. Prev Med. 2000; 30(1):43-50.

2. Airhihenbuwa CO, Kumanyika S, Agurs TD, et al. Perceptions and beliefs about exercise, rest, and health among African-Americans. Am J Health Promot. 1995; 9(6):426-9.

3. Banks-Wallace J, Conn V. Interventions to promote physical activity among African American women. Public Health Nurs. 2002; 19(5): 321-35.

4. Banks-Wallace J. Outcomes from Walk the Talk: a nursing intervention for Black women. Abnf J. 2007; 18(1):19-24.

5. Barbeau P, Johnson MH, Howe CA, et al. Ten months of exercise improves general and visceral adiposity, bone, and fitness in black girls. Obesity (Silver Spring). 2007; 15(8):2077-85.

6. Brownson RC, Eyler AA, King AC, et al. Patterns and correlates of physical activity among US women 40 years and older. Am J Public Health. 2000; 90(2):264-70.

7. Centers for Disease control and Prevention. Age-Adjusted Prevalence of Diagnosed Diabetes by Race/Ethnicity and Sex, United States, 1980-2005. 2007 [cited 2008 January 3]; Available from: http:// www.cdc.gov/diabetes/statistics/prev/national/figraceethsex.htm.

8. Centers for Disease Control and Prevention, Prevalence of regular physical activity among adults-United States, 2001 and 2005. MMWR Morb Mortal Wkly Rep. 2007; 56(46):1209-12.

9. Chlebowski RT, Pettinger M, Stefanick ML, et al. Insulin, physical activity, and caloric intake in postmenopausal women: breast cancer implications. J Clin Oncol. 2004; 22(22):4507-13.

10. Covelli MM. Efficacy of a school-based cardiac health promotion intervention program for African-American adolescents. Appl Nurs Res. 2008; 21(4):173-80.

11. Dunn AL, Marcus BH, Kampert JB, et al. Reduction in cardiovascular disease risk factors: 6-month results from Project Active. Preventive Medicine. 1997; 26(6):883-92.

12. Dunn AL, Garcia ME, Marcus BH, et al. Six-month physical activity 
and fitness changes in Project Active, a randomized trial. Medicine $\mathfrak{E}$ Science in Sports $\mathcal{E}$ Exercise. 1998; 30(7):1076-83.

13. Dunn AL, Marcus BH, Kampert JB, et al. Comparison of lifestyle and structured interventions to increase physical activity and cardiorespiratory fitness: a randomized trial [see comments]. JAMA. 1999; 281(4): 327-34.

14. Dutton GR, Davis Martin P, Welsch MA, et al. Promoting physical activity for low-income minority women in primary care. Am J Health Behav. 2007; 31(6):622-31.

15. Gaston MH, Porter GK, Thomas VG. Prime Time Sister Circles: evaluating a gender-specific, culturally relevant health intervention to decrease major risk factors in mid-life African-American women. $J$ Natl Med Assoc. 2007; 9(4):428-38.

16. Gordon-Larsen P, Nelson MC, Page P, et al. Inequality in the built environment underlies key health disparities in physical activity and obesity. Pediatrics. 2006; 117(2):417-24.

17. Hu FB, Li TY, Colditz GA, et al. Television watching and other sedentary behaviors in relation to risk of obesity and type 2 diabetes mellitus in women. Jama. 2003; 289(14):1785-91.

18. Hyman DJ, Pavlik VN, Taylor WC, et al. Simultaneous vs sequential counseling for multiple behavior change. Arch Intern Med. 2007; 167(11):1152-8.

19. Kimm SY, Barton BA, Obarzanek E, et al. Obesity development during adolescence in a biracial cohort: the NHLBI Growth and Health Study. Pediatrics. 2002; 110(5):e54.

20. Kimm SY, Glynn NW, Kriska AM, et al. Decline in physical activity in Black girls and white girls during adolescence. The New England Journal of Medicine. 2002; 347(10):709-15.

21. King AC, Haskell WL, Young DR, et al. Long-term effects of varying intensities and formats of physical activity on participation rates, fitness, and lipoproteins in men and women aged 50 to 65 years. Circulation. 1995; 91(10):2596-604.

22. King AC, Kiernan M, Oman RF, et al. Can we identify who will adhere to long-term physical activity? Signal detection methodology as a potential aid to clinical decision making. Health Psychol. 1997; 16(4): 380-9.

23. Klein DJ, Aronson Friedman L, Harlan WR, et al. Obesity and the development of insulin resistance and impaired fasting glucose in black and white adolescent girls: a longitudinal study. Diabetes Care. 2004; 27(2):378-83.

24. Koretz RL. Considerations of study design. Nutr Clin Pract. 2007; 22(6): 593-8.

25. Lara A, Yancey AK, Tapia-Conye R, et al. Pausa para tu Salud: reduction of weight and waistlines by integrating exercise breaks into workplace organizational routine. Prev Chronic Dis. 2008; 5(1):A12.

26. Last JM, Abramson JH, Friedman GD, et al., eds. A Dictionary of Epidemiology. 3rd ed. New York: Oxford University Press; 1995.

27. Lindberg R. Active living: on the road with the 10,000 Steps program. J Am Diet Assoc. 2000; 100(8):878-9.

28. Marcus BH, Dubbert PM, Forsyth LH, et al. Physical activity behavior change: issues in adoption and maintenance. Health Psychol. 2000; 19(Suppl 1):32-41.

29. Marshall SJ, Jones DA, Ainsworth BE, et al. Race/ethnicity, social class, and leisure-time physical inactivity. Med Sci Sports Exerc. 2007; 39(1):44-51.

30. McTiernan A, Kooperberg C, White E, et al. Recreational physical activity and the risk of breast cancer in postmenopausal women: the Women's Health Initiative Cohort Study. Jama. 2003; 290(10):1331-6.

31. Morrison JA, Sprecher DL, Barton BA, et al. Overweight, fat patterning, and cardiovascular disease risk factors in black and white girls: The National Heart, Lung, and Blood Institute Growth and Health Study. J Pediatr. 1999; 135(4):458-64.
32. Murrock CJ, Madigan E. Self-efficacy and social support as mediators between culturally specific dance and lifestyle physical activity. Res Theory Nurs Pract. 2008; 22(3):192-204.

33. National Center for Health Statistics, Health, United States, 2007 with Chartbook on Trends in the Health of Americans. Hyattsville, MD; 2007.

34. Orleans CT. Promoting the maintenance of health behavior change: recommendations for the next generation of research and practice. Health Psychol. 2000; 19(Suppl 1):76-83.

35. Plescia M, Herrick $\mathrm{H}$, Chavis L. Improving health behaviors in an African American community: the Charlotte Racial and Ethnic Approaches to Community Health project. Am J Public Health. 2008; 98(9):1678-84.

36. Powell LM, Slater S, Chaloupka FJ, et al. Availability of physical activity-related facilities and neighborhood demographic and socioeconomic characteristics: a national study. American Journal of Public Health. 2006; 96(9):1676-80. Epub: 2006 Jul 27.

37. Rucker-Whitaker C, Basu S, Kravitz G, et al. A pilot study of selfmanagement in African Americans with common chronic conditions. Ethn Dis. 2007; 17(4):611-6.

38. Taylor WC, Baranowski T, Young DR. Physical activity interventions in low-income, ethnic minority, and populations with disability. Am J Prev Med. 1998; 15(4):334-43.

39. Thompson D, Baranowski T, Cullen K, et al. Food, fun, and fitness internet program for girls: pilot evaluation of an e-Health youth obesity prevention program examining predictors of obesity. Prev Med. 2008; 47(5):494-7.

40. U.S. Department of Health and Human Services, Physical Activity and Health: A Report of the Surgeon General. Hyattsville, MD: U. S. Department of Health and Human Services, Centers for Disease Control and Prevention, National Center for Chronic Disease Prevention and Health Promotion; 1996.

41. Ward SA. Why we all should learn to dance: Reflecting on the African American culture. Journal of Physical Education, Recreation, and Dance. 2007; 78(5):3-5, 47-8.

42. Ward SA. African-centered Dance: An Intervention Tool in HIV/AIDS Prevention. Journal of Physical Education, Recreation, and Dance. 2008; 79(7):4-5

43. Whitt-Glover MC, Taylor WC, Heath GW, et al. Self-reported physical activity among blacks estimates from national surveys. Am J Prev Med. 2007; 33(5):412-7.

44. Whitt-Glover MC, Hogan PE, Lang W, et al. Pilot study of a faith-based physical activity program among sedentary blacks. Prev Chronic Dis. 2008; 5(2):A51.

45. Whitt-Glover MC, Kumanyika SK. Systematic review of interventions to increase physical activity and physical fitness in African-Americans. Am J Health Promot. 2009; 23(6):S33-56.

46. Whitt M, Kumanyika S, Bellamy S. Amount and Bouts of Physical Activity in a Sample of African-American Women. Med Sci Sports Exerc. 2003; 35(11):1887-93.

47. Whitt MC, DuBose KD, Ainsworth BE, et al. Walking patterns in a sample of African American, Native American, and Caucasian women: the cross-cultural activity participation study. Health Educ Behav. 2004; 31(Suppl 4):45S-56S.

48. Wilcox S, Laken M, Bopp M, et al. Increasing physical activity among church members: community-based participatory research. Am J Prev Med. 2007; 32(2):131-8.

49. Wing RR. Cross-cutting themes in maintenance of behavior change. Health Psychol. 2000; 19(Suppl 1):84-8.

50. Yancey AK, McCarthy WJ, Taylor WC, et al. The Los Angeles Lift Off: a sociocultural environmental change intervention to integrate physical activity into the workplace. Prev Med. 2004; 38(6):848-56. 